\title{
Childhood contact predicts hemispheric asymmetry in cross-race face processing
}

\author{
Megan M. Davis ${ }^{1} \cdot$ Sean M. Hudson ${ }^{2} \cdot$ Debbie S. Ma $^{3} \cdot$ Joshua Correll $^{2,4}$
}

Published online: 23 November 2015

(C) Psychonomic Society, Inc. 2015

\begin{abstract}
Participants typically process same-race faces more quickly and more accurately than cross-race faces. This deficit is amplified in the right hemisphere of the brain, presumably due to its involvement in configural processing. The present research tested the idea that cross-race contact tunes cognitive and perceptual systems, influencing this asymmetric racebased deficit in face processing. Participants with high and low levels of contact performed a lateralized recognition task with same- and cross-race faces. Replicating prior work, participants with minimal contact showed cross-race deficits in processing that were larger in the right hemisphere. For participants with more contact, this lateralized deficit disappeared. This effect of contact seems to be independent of race-based attitudes (e.g., prejudice).
\end{abstract}

Keywords Face perception $\cdot$ Face recognition $\cdot$ Social cognition

Expertise with stimuli that have a common configuration can affect processing in important ways. Though nonexperts are typically faster to classify stimuli at a basic level (e.g., naming

Electronic supplementary material The online version of this article (doi:10.3758/s13423-015-0972-7) contains supplementary material, which is available to authorized users.

Joshua Correll

joshua.correll@colorado.edu

University of Illinois, Urbana-Champaign, IL, USA

University of Colorado, Boulder, CO, USA

California State University, Northridge, CA, USA

4 Department of Psychology \& Neuroscience, UCB 345, Boulder, CO 80309-0345, USA a collie a "dog"), experts categorize exemplars from their domain of expertise (but not exemplars outside that domain) just as quickly at a subordinate level (Tanaka \& Taylor, 1991). Expertise can also increase holistic processing, such that the features of a stimulus and their arrangement are processed as a gestalt (Diamond \& Carey, 1986).

Subordinate categorization and holistic processing may both rely heavily on the right hemisphere of the brain. Individuals classify images more quickly at a basic level when stimuli are presented in the right visual field, which projects to the left hemisphere. But they classify the same images at a subordinate level more quickly when the images are presented in the left visual field, which projects to the right hemisphere (Laeng, Zarrinpar, \& Kosslyn, 2003). Perceivers also make categorical spatial judgments more quickly when stimuli appear in the right visual field, whereas they judge continuous spatial relationships (those involved in holistic processing) more quickly when stimuli are presented on the left (Kosslyn et al., 1989). Though both hemispheres can perform both kinds of processing, the left hemisphere seems to be preferentially involved in basic-level and feature-based processing, whereas the right is preferentially involved in subordinate-level and holistic processing (Hellige, Laeng, \& Michimata, 2010).

Because we have expertise with faces, face perception should rely on subordinate-level, holistic/configural, and right-lateralized processing. Indeed, perceivers swiftly classify faces at the subordinate level and more accurately recognize the parts of faces appearing in the context of the whole face rather than in isolation (Tanaka, 2001; Tanaka \& Farah, 1993). Furthermore, individuals show greater activation in the right middle fusiform gyrus, when viewing faces relative to other stimuli (Kanwisher, McDermott, \& Chun, 1997) and when matching whole relative to parts of faces (Rossion et al., 2000). 
Despite humans' considerable expertise with faces, there is variation in the types of faces that individuals encounter. This point is illustrated in the case of race. Individuals tend to have less extensive, less individuated contact with people who belong to racial out-groups. Reduced experience seemingly leads to a variety of race-based differences in categorization, processing, and hemispheric activation. Individuals typically recognize same-race better than cross-race faces, indicating greater subordinate-level processing of same-race faces (termed the cross-race recognition deficit, or CRD; see Meissner \& Brigham, 2001). They also categorize cross-race faces by race (a basic-level categorization) more quickly than same-race faces (Caldara, Rossion, Bovet, \& Hauert, 2004), and they engage in more holistic processing for same-race than for crossrace faces (Michel, Rossion, Han, Chung, \& Caldara, 2006).

These race effects on subordinate-level and holistic/ configural processing generate the prediction that race affects a perceiver's reliance on the left and right hemispheres. Correll, Lemoine, and Ma (2011; see also Turk, Handy, \& Gazzaniga, 2005) tested this prediction, recruiting Black and White participants to complete a face recognition task that involved Black and White faces. On some trials, the task presented stimuli to a participant's left visual field, projecting to the right hemisphere (LVF-rh trials); on other trials, the task presented stimuli to the right visual field, projecting to the left hemisphere (RVF-lh trials). Replicating previous research, recognition was impaired for cross-race faces. Black participants were less accurate when responding to White than to Black faces, and White participants were less accurate when responding to Black than to White faces. However, for both groups of participants, the magnitude of this CRD was especially pronounced in the LVF-rh, supporting the idea that the deficit in out-group face processing derives from the fact that in-group (but not outgroup) faces induce subordinate-level, configural, rightlateralized processing. We refer to this asymmetric deficit (a Race $\times$ Hemisphere interaction) as the CRD asymmetry.

Supporting an expertise account, experience with cross-race faces can attenuate many race-based processing differences. High-experience individuals exhibit an attenuated CRD (Chiroro \& Valentine, 1995). Similarly, providing experience via individuated training with cross-race faces improves face recognition (Lebrecht et al., 2009). Experience has also been shown to reduce racial differences in configural processing (Hancock \& Rhodes, 2008). In light of these effects, in the present experiment we explored the possibility that contact affects hemispheric asymmetries in face processing.

In sum, the CRD may stem in part from differential experience with same-race versus cross-race faces, which leads to processing differences between the two classes of stimuli. Just as increased experience with cross-race faces reduces differences in subordinate-level and configural processing, it should attenuate race-based differences in hemispheric asymmetry. In the present study, we examined the magnitude of the CRD asymmetry for White participants who reported high and low levels of contact with Blacks. We predicted that (a) participants with minimal contact should show a pronounced CRD asymmetry, but (b) participants with more extensive contact should not.

\section{Method}

\section{Participants and design}

A total of 116 University of Chicago undergraduates (82 female, 34 male; mean age $=19.34$ years ) completed an online questionnaire (for the participant recruitment details, see the Supplemental materials). Through the questionnaire, we assessed exposure to other racial groups from birth through age 18 . To oversample individuals with extensive cross-race contact, we selected 12 participants who reported (a) at least one close cross-race friend before age 6 , and (b) social networks that were at least $20 \%$ Black. We also recruited 12 respondents with minimal cross-race exposure. Eighteen additional participants were recruited via the Department of Psychology's research participation system, for a total of 42 ( 25 female, 17 male; mean age $=20.10$ years).

The participants (all White and right-handed) completed a visual half-field face recognition task in which a male to-beremembered (TBR) face (either Black or White) was presented centrally, followed by a test face of the same race. The test face was presented to either the LVF-rh or the RVF-lh. On some trials, the test face was identical to the TBR face; on other trials, the two faces differed. Thus, the task design involved a 2 (Face Race: Black vs. White) $\times 2$ (Visual Field: LVF-rh vs. RVF-lh) $\times$ 2 (Pair Correspondence: match vs. mismatch) repeated measures design. This task was administered to participants who varied continuously in terms of childhood contact with Blacks, so contact was a between-participants factor.

\section{Materials and procedure}

Photographs of 12 Black and 12 White male faces were grayscaled and equated for luminance and contrast (both within and across races) using Adobe Photoshop. An oval mask was imposed to remove the hair and clothing (see Figs. 1 and 2). The final images were $100 \times 140$ pixels.

Participants came to the laboratory twice (Times 1 and 2), separated by one week. At each session, they were greeted by a White female researcher and seated at an eMac computer with a 17 -in. screen (resolution $=800 \times 600$ ). Participants rested their chins on a chinrest positioned $18 \mathrm{in}$. from the screen and level with its center.

Recognition task Each trial began with an instruction to remember the face that would subsequently be presented. A 


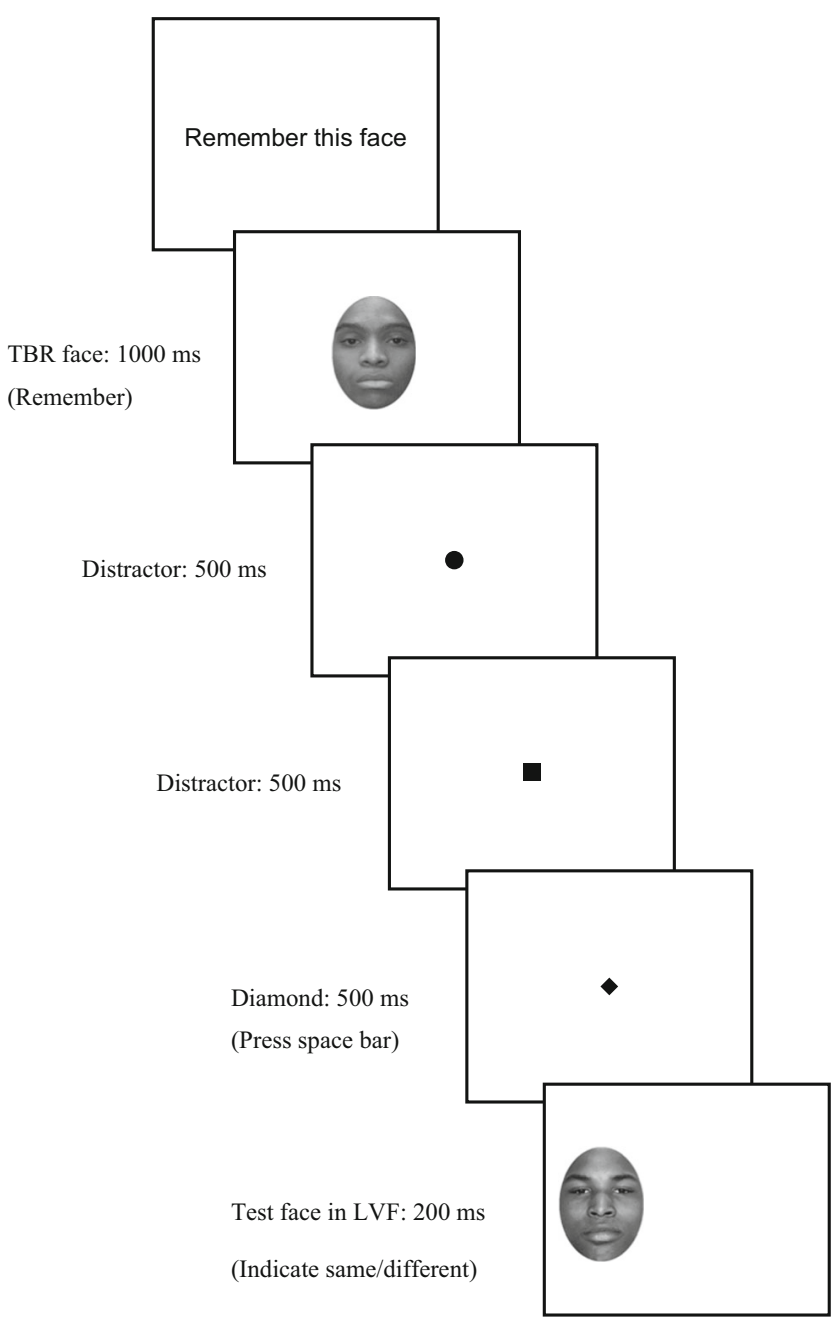

Fig. 1 Example trial from the recognition task

randomly selected TBR face was presented for $1,000 \mathrm{~ms}$ in the center of the screen. Next, to ensure attention to the center of the screen (Correll et al., 2011; Levine \& Koch-Weser, 1982), a series of two to four geometric shapes appeared at the center of the screen, each for $500 \mathrm{~ms}$. The sequence always

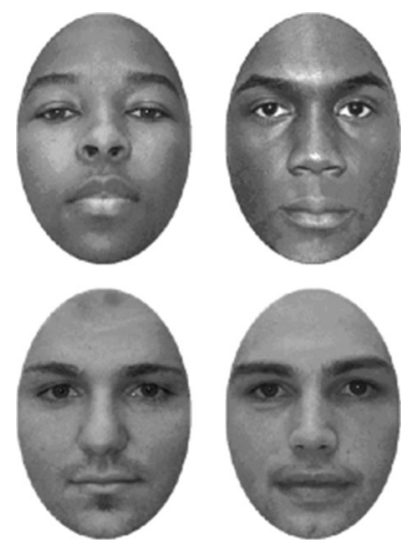

Fig. 2 Example Black and White face stimuli culminated with the presentation of a diamond. Participants were instructed to press the space bar with their left hand when the diamond appeared. Failure to respond within $500 \mathrm{~ms}$ terminated the trial. When participants responded within the time window, a randomly selected test face (always the same race as the TBR face) was immediately presented for $200 \mathrm{~ms}$ to either the LVF-rh or the RVF-lh. Display ports were centered 150 pixels, or roughly $8^{\circ}$, to the left and right of fixation. Participants were instructed to press the $\mathrm{M}$ or the $\mathrm{K}$ key with their right index or middle finger to indicate whether the test face was the same as or different from the TBR face (key assignments were counterbalanced). The task included eight practice trials, four buffer trials, and 128 test trials. This task differed slightly from previous work (Correll et al., 2011) in that it focuses on speeded response latencies rather than the accuracy of a response. Hemispheric differences have been found using both latency and error rate measures (Hellige et al., 2010), but a slower, more deliberative response allows time for the hemispheres to communicate, compromising the distinction between LVF-rh versus RVF-lh presentation and raising the possibility that condition effects might reflect something other than hemispheric differences. The use of a latency measure constitutes an effort to replicate previous findings using a paradigm that better isolates the hemispheres.

Questionnaire At Time 2, participants completed a questionnaire. Six participants were unable to return and were asked to complete the questionnaire online (four complied). The questionnaire assessed demographics, handedness, racial attitudes (with the Modern Racism Scale [MRS]: McConahay, Hardee, \& Batts, 1981; Discrimination Scale [DIS]: Wittenbrink, Judd, \& Park, 1997; and Internal \& External Motivation to Respond Without Prejudice Scales [IMS \& EMS]: Plant \& Devine, 1998), and cross-race contact. The contact questions assessed exposure to members of different racial groups before age 18 (Cloutier, Li, \& Correll, 2014). These questions instructed participants to answer six items, reporting the racial makeup of their social networks during three separate stages of childhood ( 0 to 6,6 to 12 , and 12 to 18 years), once for adults in their network and once for peers. For example, the $0-6$ peer item asked participants to consider their experiences from ages $0-6$ (described as before elementary school) and to estimate the percentage of their peers who were Asian, Black, Latino, White, and "other" (summing to $100 \%$ ).

\section{Results}

\section{Data preparation}

Recognition task We analyzed the response time data from trials on which participants responded correctly (69.66\%). The data from correct responses on which participants 
responded faster than $150 \mathrm{~ms}(3.40 \%)$ or slower than $1,500 \mathrm{~ms}$ $(3.83 \%)$ were excluded. Response times from the remaining trials were log-transformed and averaged within each cell of the Face Race $\times$ Visual Field $\times$ Pair Correspondence task design .

Questionnaire After reverse scoring, we computed scale indices for the MRS, DIS, IMS, and EMS ( $\alpha \mathrm{s}=.81, .77, .68$, and .87 , respectively). The MRS and DIS scales both measure prejudice and were correlated, $r(35)=.60$, so we combined them into a single index. We computed each participant's contact with Blacks by averaging the reported percentages of Blacks in each of the six contact items (Adult vs. Peer $\times$ Ages 0-6, 6-12, 12-18). Reported contact was highly correlated across items $(\alpha=.95)$. Despite our efforts to oversample high-contact participants, this index was also highly skewed $(M=10.27, S D=11.14$, skewness $=2.10)$. A square-root transformation reduced the skew to acceptable levels $(0.55)$. Analyses were conducted with the square-root-transformed indices, but the original variables yielded similar results.

Excluded participants Two individuals (4.76\%) failed to complete the questionnaire and were excluded from the analysis. We also excluded three participants on the basis of their task performance. Two had exceptionally fast response times $(<150 \mathrm{~ms})$ for every correct decision in multiple cells of the task; a third had slow responses $(>1,500 \mathrm{~ms})$ for every correct decision in multiple cells. Thus, we could not compute means for these individuals. One additional participant withdrew from participation. Ultimately, we had complete data from 36 White right-handed participants ( 21 female, 15 male; mean age $=20.14$ years).

\section{Primary analysis}

We analyzed response latencies as a function of Face Race $\times$ Visual Field $\times$ Pair Correspondence $\times$ Contact with Blacks. The first three factors varied within participants; the fourth factor varied continuously between participants. We predicted that the magnitude of the Face Race $\times$ Visual Field interaction would depend on contact: White participants with minimal contact should have difficulty processing Black faces in a subordinate-level, configural fashion, leading to a pronounced CRD, particularly in the LVF-rh (Correll et al., 2011). By contrast, participants with greater contact should process Black and White faces more similarly.

The predicted Face Race $\times$ Visual Field $\times$ Contact With Blacks interaction was significant, $F(1,34)=4.65, p<.039, \eta^{2}$ $=.12$ (see Figs. 3 and 4). To interpret this pattern, we examined the Face Race $\times$ Visual Field interaction separately at low and high levels of contact ( \pm 1 standard deviation). For participants with minimal contact (corresponding to a network with $1.27 \%$ Black people), we observed a significant Face Race $\times$ Visual Field interaction, $F(1,34)=6.40, p<.017, \eta^{2}=.16$. This interaction derives from the fact that, when faces were presented to

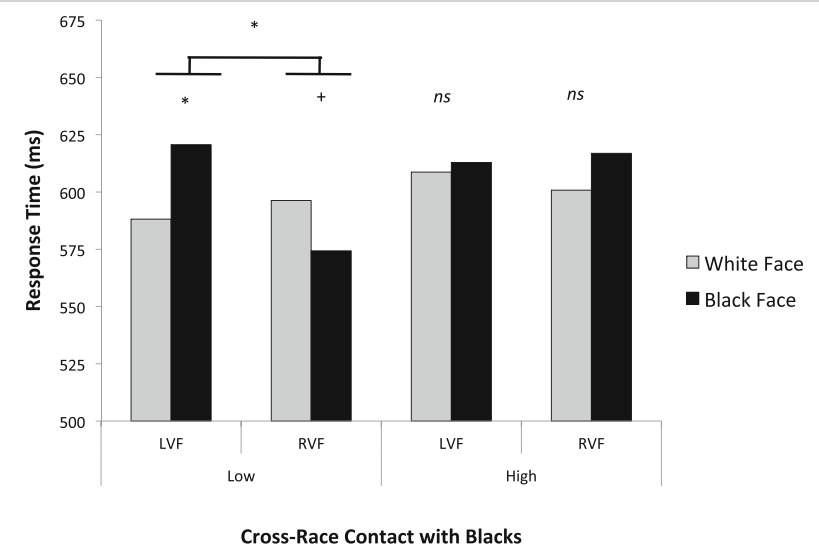

Fig. 3 Mean response times as a function of face race (Black or White), visual field (LVF or RVF), and contact with Blacks (low $=-1 S D$, high = $+1 S D)$

the LVF-rh, low-contact participants showed clear evidence of a $\mathrm{CRD}$, responding more quickly to White than to Black faces, $F(1,34)=4.97, p<.033, \eta^{2}=.13$. But when test faces were presented to the RVF-lh, low-contact participants responded (nonsignificantly) more quickly to Black than to White faces, $F(1,34)=2.82, p<.11, \eta^{2}=.08$. For high-contact participants (corresponding to a network with $19.43 \%$ Black people), we found no evidence of a Face Race $\times$ Visual Field interaction, nor was there evidence that race influenced processing in either the LVF-rh or RVF-lh, $F \mathrm{~s}(1,34)=0.29,0.07$, and $1.39 ; p \mathrm{~s}<.59$, .78 , and $.25 ; \eta^{2} \mathrm{~s}=.01, .002$, and .04 , respectively.

We anticipated a pattern of right-hemispheric dominance for same-race faces, with faster response times when faces appeared in the LVF-rh rather than the RVF-lh. No evidence of such an effect was apparent. An analysis of only the White-face trials showed no effect of visual field, $F(1,34)=0.00, p<.98$, $\eta^{2}=0$. This null effect may reflect the fact that feature-based judgments are faster overall (e.g., Leder \& Bruce, 2000). Even though it is typically less effective for recognition, RVF-lh

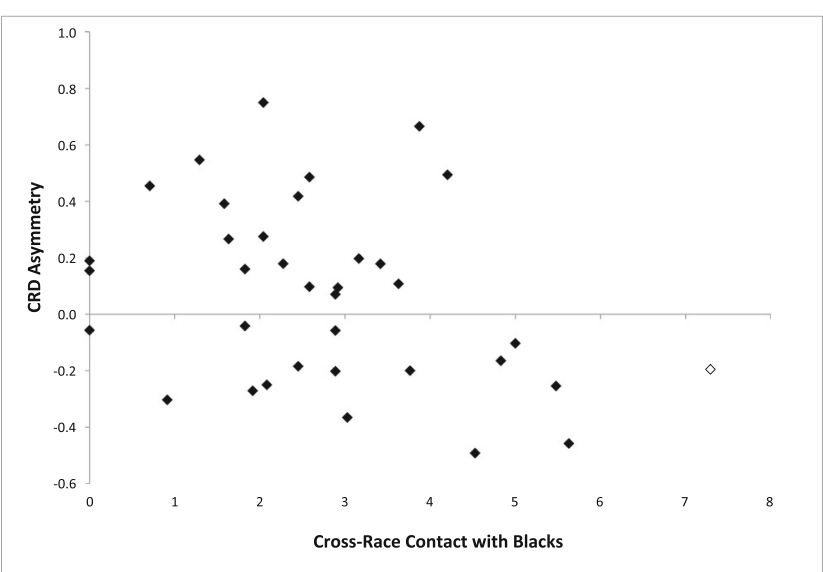

Fig. 4 Scatterplot of CRD asymmetry as a function of the square-roottransformed index of contact with Black people. Note that exclusion of the participant with the highest level of contact (designated with the hollow marker) does not alter the results 
processing may be faster, on average, masking any right-side processing advantage. Regardless, for low-contact participants, cross-race processing is dramatically impaired in the LVF-rh relative to the RVF-lh, $F(1,34)=11.56, p<.002, \eta^{2}=.25$. As predicted, low-contact participants seemed to have difficulty processing Black faces in the left visual field. By contrast, for faces with which participants have substantial contact (for all participants viewing White faces, and for high-contact participants viewing Black faces), visual field has no effect, $F \mathrm{~s}(1,34)$ $<0.34, p \mathrm{~s}>.57, \eta^{2} \mathrm{~s}<.01$.

The analysis also revealed a Face Race $\times$ Visual Field $\times$ Pair Correspondence interaction, $F(1,35)=7.79, p<.009, \eta^{2}$ $=.19$. Though we did not predict this interaction, it is not entirely surprising. The CRD asymmetry (the Face Race $\times$ Visual Field interaction) was more pronounced on mismatch than on match trials. Analyzing the two sets of trials separately, the Face Race $\times$ Visual Field interaction was significant for mismatch trials, $F(1,34)=7.09, p<.012, \eta^{2}=.17$. On match trials, it was not, $F(1,34)=0.81, p<.38, \eta^{2}=.02$, perhaps because, when the TBR and test faces were identical, participants engaged in simplistic pattern matching. However, the degree to which contact moderated the CRD asymmetry was not moderated by correspondence: The four-way interaction was not significant, $F(1,34)=0.14, p<.71, \eta^{2}=.004$.

\section{Ancillary analyses}

Demographics In a multiple regression, we predicted the CRD-asymmetry index as a function of contact with Blacks, age, gender, and conservatism (Table 1). Conservatism and gender marginally predicted the asymmetry, such that more conservative and female participants demonstrated greater

Table 1 Relationship between contact and CRD asymmetry, controlling for demographics and attitudes

\begin{tabular}{llll}
\hline Predictors & $\begin{array}{l}\text { Model 1 } \\
\text { Demographic } \\
\text { Controls }\end{array}$ & $\begin{array}{l}\text { Model 2 } \\
\text { Attitude } \\
\text { Controls }\end{array}$ & $\begin{array}{l}\text { Model 3 } \\
\text { All Controls }\end{array}$ \\
\hline Black contact & $-.39^{*}$ & $-.35^{*}$ & $-.42^{*}$ \\
Gender & .27 & & $.35^{\dagger}$ \\
Age & -.02 & & -.17 \\
Conservatism & .27 & & .36 \\
Prejudice & & .04 & -.32 \\
IMS & & .00 & -.05 \\
EMS & & .02 & .05 \\
\hline
\end{tabular}

The table shows standardized regression coefficients from multiple regressions predicting the magnitude of the CRD asymmetry as a function of contact with Black people and (1) demographic control variables, (2) attitudinal control variables, and (3) both demographic and attitudinal control variables. IMS $=$ internal motivation to respond without prejudice; $\mathrm{EMS}=$ external motivation to respond without prejudice. ${ }^{*} p<.05,{ }^{\dagger} p<.10$ asymmetries. But controlling for demographics did nothing to diminish the effect of contact.

Attitudes We similarly examined the effect of contact controlling for racial attitudes, predicting the CRD asymmetry as a function of contact, prejudice, IMS, and EMS (Table 1). None of the attitude scales predicted the asymmetry, and the contact effect remained significant. These findings undermine the possibility that our effects were driven by motivation or affect rather than contact. ${ }^{1}$

\section{Discussion}

Previous work has shown a CRD asymmetry: The CRD is more pronounced when stimuli are presented to the right hemisphere of the brain (Correll et al., 2011; Turk et al., 2005). In this study, we tested White participants' ability to recognize Black and White faces presented to either the LVFrh or RVF-lh. The results not only replicated the CRD asymmetry, they also demonstrated moderation by a theoretically important variable: childhood contact. Participants reporting minimal cross-race contact showed clear evidence of a CRD asymmetry. By contrast, participants with higher levels of contact processed Black and White faces similarly. These findings suggest that contact alters the neural processes involved in cross-race face recognition, highlighting experience-dependent flexibility. This work supports and extends research showing that contact promotes subordinate and holistic processing of out-groups (Cloutier et al., 2014).

It is possible that these effects derive from processing fluency (Gauthier, Williams, Tarr, \& Tanaka, 1998). If contact promotes efficient configural processing of Black faces, that processing may operate as a default strategy for high-contact participants. However, contact may also make the out-group more motivationally relevant, leading high- but not lowcontact participants to exert greater effort when processing Black faces (Hugenberg, Young, Bernstein, \& Sacco, 2010). The fact that the effect of contact in this study was independent of interracial motives and attitudes seems inconsistent with this possibility. Still, motivation may lead individuals with minimal experience to configurally process unfamiliar stimuli, including faces (Hugenberg \& Corneille, 2009). Richler, Wong, and Gauthier (2011) offered a potentially valuable framework for understanding these effects. They argued that whereas novices can invoke configural/holistic or rightlateralized processing through strategic attention, experts invoke such processing by default. Their argument offers a

\footnotetext{
${ }^{1}$ Information regarding our exclusion criteria, the participants, response time descriptive statistics, and analyses investigating the demographics, attitudes, specificity and timing of contact, test-retest reliability, and error rates can be viewed in the Supplemental materials.
} 
potential test of the role of perceptual fluency by suggesting that novices will be more sensitive to changes in the task context, showing evidence of configural processing in some contexts, but not others. By contrast, experts should show consistent configural processing across contexts.

It is important to note the limitations of the present experiment. First, we did not directly measure configural processing. This leaves open the possibility that the hemispheric differences might reflect other types of processing that differed across hemispheres. Future work should measure configural processing in conjunction with this task to increase confidence that the results reflect differences in configural processing. Second, because we were interested in race effects, we controlled face gender by using only male faces. Considering the processing differences between male and female faces, our results may only generalize to male faces. Finally, we only used White participants. Thus, we do not know whether our results represent a target race effect (i.e., something particular about White and Black faces) or a cross-race effect (i.e., something particular to the own-race vs. cross-race distinction). Future work should include a Black participant sample to distinguish between these two possibilities and to increase the generalizability of the results.

Although race has been characterized as a fundamental dimension of social perception, and although it has been shown to affect early aspects of face processing (Ito \& Urland, 2003), these results suggest that the impact of race depends on crossrace contact. We view these data as being in line with the possibility that contact influences which dimensions of phenotypic variability are viewed as relevant and which can be ignored (cf. Scott \& Monesson, 2009). With minimal contact, variation in same-race faces may be perceived as providing valuable information about identity, whereas variation in cross-race faces may be perceived as relatively meaningless. With greater cross-race contact, variation in both same- and cross-race faces may assume psychological relevance.

\section{References}

Caldara, R., Rossion, B., Bovet, P., \& Hauert, C. A. (2004). Event-related potentials and time course of the "other-race" face classification advantage. NeuroReport, 15, 905-910.

Chiroro, P., \& Valentine, T. (1995). An investigation of the contact hypothesis of the own-race bias in face recognition. Quarterly Journal of Experimental Psychology, 48, 879-894.

Cloutier, J., Li, T., \& Correll, J. (2014). The impact of childhood experience on amygdala response to perceptually familiar Black and White faces. Journal of Cognitive Neuroscience, 26, 1992-2004.

Correll, J., Lemoine, C., \& Ma, D. S. (2011). Hemispheric asymmetry in cross-race face recognition. Journal of Experimental Social Psychology, 47, 1162-1166.

Diamond, R., \& Carey, S. (1986). Why faces are and are not special: An effect of expertise. Journal of Experimental Psychology: General, 115, 107-117. doi:10.1037/0096-3445.115.2.107
Gauthier, I., Williams, P., Tarr, M. J., \& Tanaka, J. (1998). Training "Greeble" experts: A framework for studying expert object recognition processes. Vision Research, 38, 2401-2428.

Hancock, K. J., \& Rhodes, G. (2008). Contact, configural coding and the other-race effect in face recognition. British Journal of Psychology, 99, 45-56.

Hellige, J. B., Laeng, B., \& Michimata, C. (2010). Processing asymmetries in the visual system. In K. Hugdahl \& R. Westerhausen (Eds.), The two halves of the brain: Information processing in the cerebral hemispheres (pp. 379-415). Cambridge, MA: MIT Press.

Hugenberg, K., \& Corneille, O. (2009). Holistic processing is tuned for in-group faces. Cognitive Science, 33, 1173-1181.

Hugenberg, K., Young, S. G., Bernstein, M. J., \& Sacco, D. F. (2010). The categorization-individuation model: An integrative account of the other-race recognition deficit. Psychological Review, 117, 11681187.

Ito, T. A., \& Urland, G. R. (2003). Race and gender on the brain: Electrocortical measures of attention to race and gender of multiply categorizable individuals. Journal of Personality and Social Psychology, 85, 616-626.

Kanwisher, N., McDermott, J., \& Chun, M. M. (1997). The fusiform face area: A module in human extrastriate cortex specialized for face perception. Journal of Neuroscience, 17, 43024311.

Kosslyn, S. M., Koenig, O., Barrett, A., Cave, C. B., Tang, J., \& Gabrieli, J. D. E. (1989). Evidence for two types of spatial representations: Hemispheric specialization for categorical and coordinate relations. Journal of Experimental Psychology: Human Perception and Performance, 15, 723-735. doi:10. 1037/0096-1523.15.4.723

Laeng, B., Zarrinpar, A., \& Kosslyn, S. M. (2003). Do separate processes identify objects as exemplars versus members of basic-level categories? Evidence from hemispheric specialization. Brain and Cognition, 53, 15-27.

Lebrecht, S., Pierce, L. J., Tarr, M. J., \& Tanaka, J. W. (2009). Perceptual other-race training reduces implicit racial bias. PLOS ONE, 4, e4215. doi:10.1371/journal.pone. 0004215

Leder, H., \& Bruce, V. (2000). When inverted faces are recognized: The role of configural information in face recognition. Quarterly Journal of Experimental Psychology, 53A, 513536.

Levine, S. C., \& Koch-Weser, M. P. (1982). Right hemisphere superiority in the recognition of famous faces. Brain and Cognition, $1,10-22$.

McConahay, J. B., Hardee, B. B., \& Batts, V. (1981). Has racism declined in America? It depends on who is asking and what is being asked. Journal of Conflict Resolution, 25, 563-579.

Meissner, C. A., \& Brigham, J. C. (2001). Thirty years of investigating the own-race bias in memory for faces: A meta-analytic review. Psychology, Public Policy, and Law, 7, 3-35. doi:10.1037/10768971.7.1.3

Michel, C., Rossion, B., Han, J., Chung, C. S., \& Caldara, R. (2006). Holistic processing is finely tuned for faces of one's own race. Psychological Science, 17, 608-615.

Plant, E. A., \& Devine, P. G. (1998). Internal and external motivation to respond without prejudice. Journal of Personality and Social Psychology, 75, 811-832.

Richler, J. J., Wong, Y. K., \& Gauthier, I. (2011). Perceptual expertise as a shift from strategic interference to automatic holistic processing. Current Directions in Psychological Science, 20, 129-134. doi:10. 1177/0963721411402472

Rossion, B., Dricot, L., Devolder, A., Bodart, J. M., Crommelinck, M., De Gelder, B., \& Zoontjes, R. (2000). Hemispheric asymmetries for whole-based and part-based face processing in the human fusiform 
gyrus. Journal of Cognitive Neuroscience, 12, 793-802. doi:10. 1162/089892900562606

Scott, L. S., \& Monesson, A. (2009). The origin of biases in face perception. Psychological Science, 20, 676-680.

Tanaka, J. W. (2001). The entry point of face recognition: Evidence for face expertise. Journal of Experimental Psychology: General, 130, 534-543. doi:10.1037/0096-3445.130.3.534

Tanaka, J. W., \& Farah, M. J. (1993). Parts and wholes in face recognition. Quarterly Journal of Experimental Psychology, 46A, 225-245. doi:10.1080/14640749308401045
Tanaka, J. W., \& Taylor, M. (1991). Object categories and expertise: Is the basic level in the eye of the beholder? Cognitive Psychology, 23, 457-482.

Turk, D. J., Handy, T. C., \& Gazzaniga, M. S. (2005). Can perceptual expertise account for the own-race bias in face recognition? A splitbrain study. Cognitive Neuropsychology, 22, 877-883.

Wittenbrink, B., Judd, C. M., \& Park, B. (1997). Evidence for racial prejudice at the implicit level and its relationship with questionnaire measures. Journal of Personality and Social Psychology, 72, 262274. 